\title{
Rabattverträge sichern Verordnungsfähigkeit
}

- Das lange Tauziehen um die Verordnungs- und Erstattungsfähigkeit des lang wirksamen Insulinanalogons Insulinglargin ist entschieden. Über den Weg von Rabattverträgen bleiben sie GKV-Patienten in der Routineversorgung erhalten.

Mit einem hohen Anteil an den Gesundheitskosten in Deutschland und täglich rund 1000 Neuerkrankungen ist Typ-2-Diabetes eine erhebliche Belastung für die Volkswirtschaft. Entsprechend rückt die Wirtschaftlichkeit von Therapiestrategien immer mehr in den Vordergrund. ,Wobei sich alle einig sind, dass ein besseres Produkt auch einen höheren Preis hat", sagte Prof. Oliver Schöffski, Nürnberg. Uneinigkeit bestünde hingegen darin, was unter einem besseren Produkt zu verstehen ist. Dies könne zu einem zähen Ringen von Pharmaunternehmen und den Bundesinstitutionen führen, wie am Beispiel der lang wirksamen Insulinanaloga deutlich wurde.

Das Institut für Qualität und Wirtschaftlichkeit im Gesundheitswesen (IQWiG) hat für Insulinglargin (Lantus ${ }^{\circledR}$ ) bezüglich des Hypoglykämierisikos einen Vorteil gegenüber anderen lang wirksamen Insulinen anerkannt. Dies führte jedoch nicht dazu, einen Anspruch auf den vom Unternehmen angestrebten Preis pro definierte Tagesdosis von 2,82 Euro (Glargin) im Vergleich zu 1,25 Euro (NPHInsulin) zu rechtfertigen. Vielmehr gelangte der $\mathrm{Ge}$ meinsame Bundesausschuss (GBA) am 18.3.2010 zu dem Beschluss, dass Insulinanaloga nicht zulasten der GKV verordnungsfähig sind, solange sie im Vergleich zu NPH-Insulin Mehrkosten erzeugen. „Für diese Entscheidung wurden nur die reinen Arznei- mittelkosten herangezogen, die Therapiekosten ignoriert und die Studien, die dazu eingereicht wurden, nicht einmal erwähnt“, empörte sich Schöffski und verwies darauf, dass zu Glargin ein umfangreiches pharmaökonomisches Studienprogramm vorliege, das überzeugend die Wirtschaftlichkeit dieser Therapie dokumentiere.

Aufgrund dieser Unstimmigkeiten bat das Bundesministerium für Gesundheit (BMG) mit Schreiben vom 21.5.2010 den GBA um eine ergänzende Stellungnahme, die am 21.6.2010 erfolgte. In ihr wird vornehmlich Kritik an den Studien geübt, die Wirtschaftlichkeit aus juristischer Sicht betont und zudem bereits bestehende Mehrwertverträge mit Krankenkassen kritisiert. Mit einem Schreiben vom 25.6.2010 teilt das BMG mit, dass es nach wie vor Sache der Krankenkassen sei, über Wertverträge die Arzneikosten zu regulieren. Aber der GBABeschluss als solcher, so Schöffski, werde nicht grundsätzlich angezweifelt oder aufgehoben. „Rabattverträge mit den Kassen bleiben jedoch weiterhin erlaubt.“

In der Zwischenzeit hat der Hersteller mit nahezu allen Krankenkassen Rabattverträge abgeschlossen (Liste unter: www.sanofi-aventis.de). ,95\% aller GKV-Patienten können die Therapie mit Glargin fortsetzen", so Schöffski. Denn über die Rabattverträge bleibt Glargin trotz GBA-Beschluss weiterhin verordnungs- und erstattungsfähig.

cvs

Pressekonferenz zur DDG-DAG-Tagung am 02.11.2010 in Berlin, Veranstalter: Sanofi-Aventis

\section{Umstellungsbedarf ist nun gegeben Alternativen zum Glitazoneinsatz}

- Das Aus für die Glitazone produziert in der Praxis Handlungsbedarf. Aber welche Alternativen kommen für betroffene Patienten in Frage?

Am 3. November hat das Bundesgesundheitsministerium dem Beschluss des GBA zur Verordnungsfähigkeit der Glitazone zugestimmt. Rosiglitazon darf nicht mehr verordnet werden - es wurde allerdings schon im September vom BfArM zurückgerufen. Pioglitazon kann nach GBA-Ansicht nur noch in medizinisch begründeten Einzelfällen und mit Begründung verordnet werden, so Dr. Hans-Martin Reuter aus Jena. Die Regelung für Pioglitazon kann aber frühestens am 1.4.2011 in Kraft treten.

Hintergrund sind unter anderem Daten, die für Rosiglitazon ein erhöhtes kardiovaskuläres Risiko zeigten. Eine Studie ergab zudem für Rosiglitazon ein höheres Risiko für Schlaganfall, Herzinsuffizienz und Tod als für Pioglitazon. Der GBA erklärte, es gebe für alle Therapiesituationen Alternativen, für die ein ähnliches Schadenspotenzial nicht bekannt sei. So sei etwa für Gliptine in Studien bis zu zwei Jahren kein relevantes Schädigungspotenzial ermittelt worden.

Patienten mit Glitazontherapie können individuell, je nach Vortherapie, umgestellt werden, so Reuter. Als Monotherapie stehen statt des Glitazons Metformin, Sulfonylharnstoff (SH), Acarbose, Sitagliptin oder Insulin zur Verfügung. Oder Pioglitazon als Reserve, vielleicht bei Unverträglichkeit von Acarbose. Aber: „Wir wissen noch nicht, was für Ausnahmen wir haben." Bei SHen und Insulin ist die Gefahr von Hyperglykämie und Gewichtszu- 